\title{
Graphical description of unitary transformations on hypergraph states
}

\author{
Mariami Gachechiladze ${ }^{1}$, Nikoloz Tsimakuridze ${ }^{2}$, \\ and Otfried Gühne ${ }^{1}$ \\ ${ }^{1}$ Naturwissenschaftlich-Technische Fakultät, Universität Siegen, \\ Walter-Flex-Straße 3, 57068 Siegen, Germany \\ ${ }^{2}$ School of Mathematics and Computer Science, Free University of Tbilisi, \\ 240 David Agmashenebeli alley, 0159 Tbilisi, Georgia
}

\begin{abstract}
Hypergraph states form a family of multiparticle quantum states that generalizes cluster states and graph states. We study the action and graphical representation of nonlocal unitary transformations between hypergraph states. This leads to a generalization of local complementation and graphical rules for various gates, such as the CNOT gate and the Toffoli gate. As an application, we show that already for five qubits local Pauli operations are not sufficient to check local equivalence of hypergraph states. Furthermore, we use our rules to construct entanglement witnesses for three-uniform hypergraph states.
\end{abstract}

\section{Introduction}

Due to its possible applications in quantum information processing, multiparticle entanglement is under intensive research. One of the problems in this field is the identification of families of states which are useful in applications, but nevertheless can be described by a simple formalism. An interesting class of multi-qubit quantum states are graph states [1. Mathematically, these states are described by graphs, where the vertices correspond to particles and the edges represent two-body interactions in a possible generation process. A generalization of these states are hypergraph states [2 5$]$. In a hypergraph, an edge can connect more than two vertices, so hypergraph states can be generated with multi-qubit interactions. Hypergraph states have turned out to violate local realism in a robust manner [6], they play a role in quantum algorithms [7] and are central for novel schemes of measurement-based quantum computation [8].

A general feature of graph and hypergraph states is that different graphs may lead to quantum states with the same entanglement properties. It is therefore important to study the action of local and nonlocal unitary transformations between these states. For graph states, the so-called local complementation plays an outstanding role [9]: This graphical transformation corresponds to so-called local Clifford operations and these operations represent all possible local unitary transformations between graph states 
for up to eight qubits [10], only for large qubit numbers other transformations play a role 11,12 .

In this paper we derive graphical rules to represent various unitary transformations between hypergraph states. First, we introduce a generalization of local complementation to hypergraphs and the corresponding unitary transformations. Then we consider different quantum gates, such as the CNOT and Toffoli gate and their graphical representation. In general, the considered unitary transformations are nonlocal, but in some cases they can be combined to give effectively local transformations. With that, we find pairs of five-qubit hypergraph states, which are equivalent under local unitary transformations, but they are not equivalent under local Pauli operations. These are the first examples of this kind, up to four qubits all locally equivalent hypergraph states could be transformed into each other by application of Pauli matrices only [5, 13]. As a second application, we construct entanglement witnesses for hypergraph states which contain only three-edges. This will be useful for characterizing entanglement in these states experimentally.

\section{Local complementation of hypergraph states}

\subsection{Basic definitions and local complementation of graphs}

Let us start by defining hypergraph states, a detailed discussion of their properties can be found in Ref. [5]. A hypergraph $H=(V, E)$ consists of a set of vertices $V=\{1, \ldots, N\}$ and a set of hyperedges $E \subset 2^{V}$, with $2^{V}$ being the power set of $V$, some examples of hypergraphs can be found in Fig. 1. While for graphs edges connect exactly two vertices, hyperedges can connect more than two vertices, or contain just a single vertex. For any hypergraph the corresponding hypergraph state $|H\rangle$ is defined as the $N$-qubit state

$$
|H\rangle=\prod_{e \in E} C_{e}|+\rangle^{\otimes N}
$$

where $|+\rangle=(|0\rangle+|1\rangle) / \sqrt{2}$ are the initial single-qubit states, $e \in E$ is a hyperedge and $C_{e}$ is a multi-qubit phase gate $C_{e}=\mathbb{1}-2|1 \ldots 1\rangle\langle 1 \ldots 1|$, acting on the Hilbert space associated with the vertices $v \in e$. Since all these phase gates commute, the order in the product does not matter. It is useful to note that hypergraph states are exactly the states that can be written as

$$
|H\rangle=\sum_{x \in\{0,1\}^{n}}(-1)^{f(x)}|x\rangle,
$$

with $f(x) \in\{0,1\}$ being some binary function. From this representation, one recognizes that hypergraph states are special cases of locally maximally entanglable (LME) states. LME states are wider classes of states as they allow arbitrary equal complex phases (corresponding to an arbitrary $f(x)$ ) in the full computational basis. The name LME is due to the fact that they are maximally entangleable to auxiliary systems using only local operations [2]. 
A very important subclass of hypergraph states are graph states. Their properties and applications have been studied extensively. Graph states correspond to graphs and therefore, only two-body controlled phase gates $C_{\{i j\}}$ are required for their generation. They are local stabiliser states and prominent examples of them are Greenberger-HorneZeilinger states and cluster states. For a review on graphs states we direct the reader to Ref. [1].

Once it is established that graph states are important classes of multiqubit states, it is crucial to learn which of these states are equivalent under local actions of each party. As local actions one considers here local unitary transformations. Here the discrete subclass of local Clifford operations play an outstanding role. By definition, local Clifford operations leave the set of Pauli matrices invariant. It has been shown that a graph state $|G\rangle$ can be transformed to another graph state $\left|G^{\prime}\right\rangle$ by means of local Clifford action on some parties, if the graph $G^{\prime}$ can be obtained from the graph $G$ by a series of local complementations [9]. The local complementation of a graph $G$ works as follows: One picks a vertex $a \in V$ and complements then subgraph in the neighbourhood $\mathcal{N}(a)$ of $a$, defined as the set containing all adjacent vertices to $a$. The complementation means that vertices in the neighbourhood become disconnected, if they were connected before, and they become connected, if they were disconnected before. Originally, the rule of local complementation has been conjectured to be necessary and sufficient for local unitary equivalence of graph states [9], however, it was later disproved by counterexamples 11,12 .

In order to physically achieve the local unitary transformation corresponding local complementation, the following unitary transform is considered:

$$
\tau_{g}(a)={\sqrt{X_{a}}}^{ \pm} \prod_{b \in N(a)}{\sqrt{Z_{b}}}^{\mp}
$$

where $X$ and $Z$ denote the Pauli matrices, and

$$
\sqrt{X}^{ \pm}=\left(\begin{array}{cc}
\frac{1 \pm i}{2} & \frac{1 \mp i}{2} \\
\frac{1 \mp i}{2} & \frac{1 \pm i}{2}
\end{array}\right)=|+\rangle\langle+| \pm i|-\rangle\langle-|, \quad \sqrt{Z^{ \pm}}=\left(\begin{array}{cc}
1 & 0 \\
0 & \pm i
\end{array}\right) .
$$

In the next section, we will generalize this to hypergraph states.

\subsection{Local complementation of hypergraphs}

Now we extend the term local complementation and its action to all hypergraph states. We denote the set of vertices of a hypergraph to be $V$ and the set of edges to be $E$. First we introduce a term which can be regarded as a generalization of the term neighbourhood known in graph theory. We call it adjacency of a vertex $a \in V$ and denote it by $\mathcal{A}(a)=\{e-\{a\} \mid e \in E$ with $a \in e\}$. The elements of $\mathcal{A}(a)$ are sets of vertices which are adjacent to $a$ via some hyperedge. To give an an example, the adjacency of the vertex $a=1$ from the hypergraph in the top image of Fig. 1 (a) is given by $\mathcal{A}(1)=\{\{3\},\{2,3\},\{4,5\}\}$. Similarly we can define the adjacency for some set of vertices $W \subseteq V$ as $\mathcal{A}(W)=\{e-W \mid e \in E$ with $W \subseteq e\}$. 
For formulating our main result, we introduce the concept of a local edge-pair complementation in hypergraphs around a vertex $a \in V$. Let us define first the set of adjacency pairs of vertex $a$ to be the set $\mathcal{A}_{2}(a)=\left\{\left\{e_{1}, e_{2}\right\} \mid e_{1} \neq e_{2}, e_{1} \in\right.$ $\left.\mathcal{A}(a), e_{2} \in \mathcal{A}(a)\right\}$ of all distinct pairs in the adjacency set. Considering again the top image from the Fig. 1 (a), the set of adjacency pairs for vertex $a=1$ is given by the set $\mathcal{A}_{2}(1)=\{\{\{3\},\{2,3\}\},\{\{3\},\{4,5\}\},\{\{2,3\},\{4,5\}\}\}$. Finally, the local edge-pair complementation around a vertex a complements the edges in the multiset $P=\left\{e_{1} \cup e_{2} \mid\left\{e_{1}, e_{2}\right\} \in \mathcal{A}_{2}(a)\right\}$. Notice that $P$ is a multiset and only the edges appearing with odd multiplicity will be affected. We again consider the top image from Fig. 1 (a) for which the multiset is $P=\{\{2,3\},\{3,4,5\},\{2,3,4,5\}\}$. Complementation of the edges in this multiset means that they are deleted from the hypergraph, if they were already present, and the are added, if they were not present.

In the following theorem we show that a local edge-pair complementation transforming a hypergraph state $|H\rangle$ to a hypergraph state $\left|H^{\prime}\right\rangle$ around a vertex $a$ can be achieved by the the following nonlocal operation:

$$
\tau(a)={\sqrt{X_{a}}}^{ \pm} \prod_{e \in \mathcal{A}(a)}{\sqrt{C_{e}}}^{\mp}
$$

where ${\sqrt{C_{e}}}^{ \pm}=\mathbb{1}-[1-( \pm i)]|11 \ldots 1\rangle\langle 11 \ldots 1|$ is the square root of controlled phase gate applied to qubits in edge $e$. It is a diagonal operator with every eigenvalue being one, except for the eigenvalue corresponding to state $|1\rangle^{\otimes|e|}$, which is $\pm i$. In the following, we sometimes write $\tau^{ \pm}(a)$ in order to indicate the sign of ${\sqrt{X_{a}}}^{ \pm}$. Note that for a usual graph Eq. (5) corresponds to Eq. (3) and local edge-pair complementation corresponds to the local complementation. We can formulate:

Theorem 1. For any hypergraph state the transformation $\tau(a)$ around a vertex $a \in V$ performs a local edge-pair complementation on its corresponding hypergraph.

Proof. Without loss of generality we assume that $a=N$ is the last qubit. Then, we need to fix the following notation. First, let $x$ or $|x\rangle$ be an element of the computational basis on the first $N-1$ qubits, $x$ can be seen as a string of 0 and 1 . We say that an edge $e$ acts on $x$ if $x$ has the entries 1 on the qubits belonging to $e$. This means that the phase gate $C_{e}$ changes the sign of $|x\rangle$. We define $m_{x}=\mid\{e \in H \mid e$ acts on $x\} \mid$ as the number edges acting on $x$. Since $x$ is defined on $N-1$ qubits only, we also define $m_{x}^{N}=\mid\left\{e \in H \mid e\right.$ acts on $\left.\left\{x, 1_{N}\right\}\right\} \mid$ as the number of edges that act on $\left\{x, 1_{N}\right\}$. This means that the edges act on the basis element, where a 1 is appended as the state of the last qubit.

Using this notation, the hypergraph state can be rewritten as: $|H\rangle=$ 


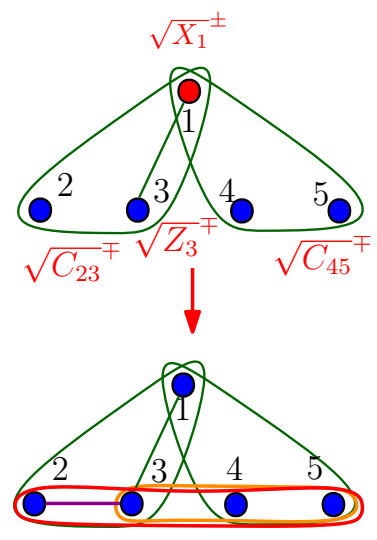

(a)

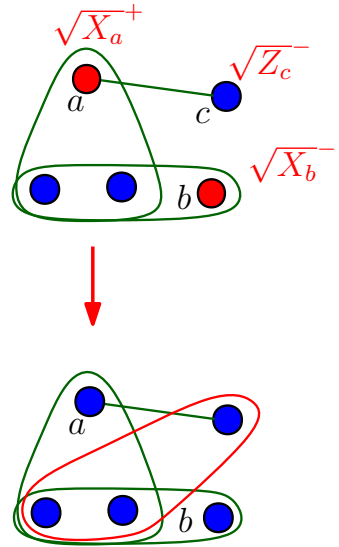

(b)

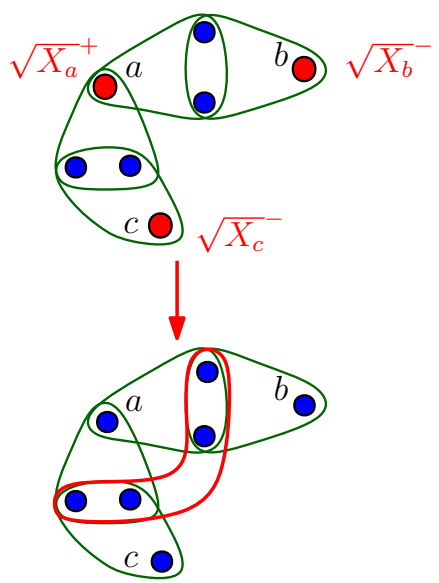

(c)

Figure 1. The top row shows hypergraphs before application of the extended local complementation rules. The bottom row shows resulting hypergraphs after the transformation has been made. (a) An example of a hypergraph state. Here set of vertices are $\{1,2,3,4,5\}$, the set of edges are $E=\{\{1,2,3\},\{1,3\},\{1,4,5\}\}$, the set of adjacencies for qubit 1 is $\mathcal{A}(1)=\{\{3\},\{2,3\},\{4,5\}\}$ and the adjacency pairs are $\mathcal{A}_{2}(1)=\{\{\{3\},\{2,3\}\},\{\{3\},\{4,5\}\},\{\{2,3\},\{4,5\}\}\}$ Therefore, the set of complemented edges are $P=\{\{2,3\},\{3,4,5\},\{2,3,4,5\}\}$. After application of $\tau(1)$ the edges from the multiset $P$ are complemented and in this case all three new edges are created. (b) Here, we apply two transformations, $\tau^{+}(a)$ and $\tau^{-}(b)$. As a consequence, the square roots of the two-qubit phase gates cancel out and we are left with local Clifford operations. This example demonstrates that local Pauli operations are not enough to exhaust all equivalence classes already in five-qubit hypergraph states. (c) An example of application of three transformations. Again, phase gates on the neighbourhoods of vertices $a, b$, and $c$ cancel out and a local transformation remains.

$\sum_{x}(-1)^{m_{x}}\left[|x\rangle \otimes\left(|0\rangle+(-1)^{m_{x}^{N}}|1\rangle\right)\right]$ and we can compute:

$$
\begin{aligned}
\tau^{+}(N)|H\rangle & ={\sqrt{X_{N}}}^{ \pm} \prod_{e \in \mathcal{A}(N)}{\sqrt{C_{e}}}^{\mp}|H\rangle \\
& ={\sqrt{X_{N}}}^{ \pm} \sum_{x}(-1)^{m_{x}}(\mp i)^{m_{x}^{N}}\left[|x\rangle \otimes\left(|0\rangle+(-1)^{m_{x}^{N}}|1\rangle\right)\right] \\
& =\sum_{x}(-1)^{m_{x}}(\mp i)^{m_{x}^{N}}( \pm i)^{m_{x}^{N} \bmod 2}\left[|x\rangle \otimes\left(|0\rangle+(-1)^{m_{x}^{N}}|1\rangle\right)\right] \\
& =\sum_{x}(-1)^{m_{x}}(\mp i)^{m_{x}^{N}-\left(m_{x}^{N} \bmod 2\right)}\left[|x\rangle \otimes\left(|0\rangle+(-1)^{m_{x}^{N}}|1\rangle\right)\right] \\
& =\sum_{x}(-1)^{m_{x}}(-1)^{\left(\begin{array}{c}
m_{x}^{N} \\
2
\end{array}\right)}\left[|x\rangle \otimes\left(|0\rangle+(-1)^{m_{x}^{N}}|1\rangle\right)\right] .
\end{aligned}
$$

Eq. (6) shows that the sign flip of $|x\rangle \otimes\left(|0\rangle+(-1)^{m_{x}^{N}}|1\rangle\right.$ is defined by $\left(\begin{array}{c}m_{x}^{N} \\ 2\end{array}\right)$. This is nothing but the number of pairs of edges in $\mathcal{A}(N)$ that act on $x$. This sign flip is equivalently described, if we apply the $C_{e}$ for all the edges $e \in P$ to the hypergraph. As $C_{e}^{2}=\mathbb{1}$, this means that edges in multiset $P$ get complemented. 
Some examples for the application of this rule are given in Fig. 1. Note that the map is not always local, since it contains ${\sqrt{C_{e}}}^{ \pm}$gates that are nonlocal whenever the vertex $a$ is contained in at least one edge of cardinality three or more. Thus, this map can change the entanglement properties of the state it is applied to. However, in particular structures of hypergraphs, the map can be chosen to be applied to multiple vertices in a way that the nonlocal gates cancel each other out. Whenever the nonlocal gates cancel each other out we can perform the complementation operation without applying those canceling gates at all. Thus the resulting hypergraph will be obtained by using local operators only.

Fig. 1(b) and (c) display two examples where a sequence of local complementations can be implemented using only local operators. These are the first examples that demonstrate that two hypergraphs, with edges containing more than two qubits, can be equivalent under local unitary operators but not under local Pauli operators. Finally, it should be noted that our rule of local complementation can also be derived from the general theory given recently in Ref. [12], but our proof is significantly simpler.

\section{Permutation unitaries and their applications}

In the previous section we considered the extension of local complementation for hypergraph states. In this section we investigate a different family of unitary transformations, we call them permutation unitaries. These transformations permute the vectors of the computational basis. Such permutations are obviously unitary and from Eq. (2) it is clear that they map hypergraph states to hypergraph states, so there must be a graphical description.

The simplest example of such a permutation unitary is Pauli-X (or NOT) gate, whose action on a hypergraph state was studied before [4, 5], see also Fig. 2 for an example. A nonlocal example of a permutation unitary in two dimensions is a CNOT gate, $\mathrm{CNOT}_{a b}:|10\rangle \leftrightarrow|11\rangle$. An extension to three-qubit is the Toffoli gate, $\mathrm{CCNOT}_{a b c}:|110\rangle \leftrightarrow|111\rangle$. Clearly, is is not necessary to consider all permutations, as for instance any permutation can be viewed as a sequence of transpositions [14. Another possibility is to look for extensions of CNOT gates. For two-qubit permutations considering one can easily see that NOT and CNOT are enough to cover all possible permutations. Additionally, it is known that every permutation on $\{0,1\}^{N}$ can be realized by means of a reversible circuit using the NOT, CNOT and CCNOT basis and at most one ancilla bit [15]. It is possible to derive a graphical rule of how such maps transform hypergraph states. Here we give rules explicitly only for the two-qubit CNOT and its multiqubit extensions, but the methodology can be applied to derive any arbitrary permutation unitary if the exact graphical transformation is needed.

Lemma 2. Applying the CNOT $T_{c t}$ gate on hypergraph state, where $c$ is the control qubit and $t$ is the target one, introduces/deletes the edges of the form $E_{t}=\left\{e_{t} \cup\{c\} \mid e_{t} \in \mathcal{A}(t)\right\}$.

Proof. Without loss of generality we assume that $\mathrm{CNOT}_{12}$ acts on the first two qubits. 


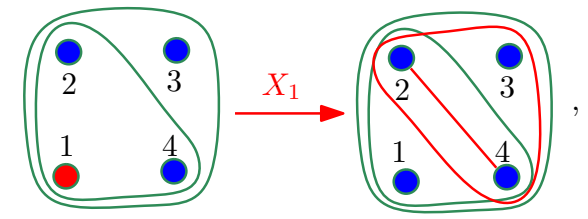

(a)

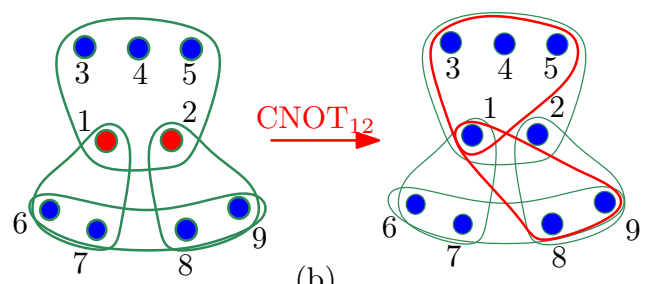

(b)

Figure 2. (a) An example of application of Pauli-X or NOT gate on the first qubit. We have $\mathcal{A}=\{\{2,4\},\{2,3,4\}\}$. (b) An example of application of $\mathrm{CNOT}_{12}$, where the first qubit is the control qubit and the second qubit is the target. For the graphical action, we have to consider $\mathcal{A}(2)=\{\{1,3,4,5\},\{8,9\}\}$ and in each of its elements we have to add the qubit 1 . So, the edges $E_{t}=\{\{1,3,4,5\},\{1,8,9\}\}$ are added (or removed, if they were already present).

We write a hypergraph state as follows:

$$
\begin{array}{rrr}
|H\rangle=|00\rangle\left|H\left(E_{00}\right)\right\rangle & E_{00}=\{e \mid e \in E, e \cap c=\emptyset, e \cap t=\emptyset\}, \\
+|01\rangle\left|H\left(E_{00}+E_{01}\right)\right\rangle & E_{01}=\{e \mid e \in \mathcal{A}(t), e \cap c=\emptyset\}, \\
+|10\rangle\left|H\left(E_{00}+E_{10}\right)\right\rangle & E_{10}=\{e \mid e \in \mathcal{A}(c), e \cap t=\emptyset\}, \\
+|11\rangle\left|H\left(E_{00}+E_{01}+E_{10}+E_{11}\right)\right\rangle & E_{11}=\{e \mid e \in \mathcal{A}(\{c, t\})\} .
\end{array}
$$

The $\mathrm{CNOT}_{12}$ gate swaps $|10\rangle$ and $|11\rangle$, or alternatively Eq. (9) and Eq. (10), but leaves the other parts invariant. Therefore we obtain the following:

$$
\begin{array}{rll} 
& E_{00}^{\text {new }}=E_{00} . \\
E_{00}^{\text {new }}+E_{01}^{\text {new }}=E_{00}+E_{01} & \Rightarrow \quad E_{01}^{\text {new }}=E_{01} . \\
E_{00}^{\text {new }}+E_{10}^{\text {new }}=E_{00}+E_{01}+E_{10}+E_{11} \quad \Rightarrow \quad & E_{10}^{\text {new }}=E_{01}+E_{10}+E_{11} . \\
E_{00}^{\text {new }}+E_{01}^{\text {new }}+E_{10}^{\text {new }}+E_{11}^{\text {new }}=E_{00}+E_{10} & \Rightarrow \quad E_{11}^{\text {new }}=E_{11} .
\end{array}
$$

Equations (11) 14) show that only the edges containing the control qubit can appear or disappear. More precisely, Eq. (13) shows that the new edges that are added/deleted are of the form $E_{t}=\left\{e_{t} \cup c \mid e_{t} \in \mathcal{A}(t)\right\}$.

An example of this rule is shown in Fig. 2. We can directly generalize this rule to extended CNOT gates, such as the Toffoli gate, the proof is essentially the same.

Corollary 3. Applying the extended $C N O T_{C t}$ gate on a hypergraph state, where a set of control qubits $C$ controls the target qubit $t$, introduces or deletes the set of edges $E_{t}=\left\{e_{t} \cup C \mid e_{t} \in \mathcal{A}(t)\right\}$.

Moreover, as mentioned above every permutation can be constructed using NOT, CNOT, and CCNOT and at most one ancilla qubit. An ancilla qubit is necessary to construct the multiqubit gate set, $\mathcal{T}=\left\{\mathrm{C}^{0} \mathrm{NOT}, \mathrm{CNOT}, \ldots, \mathrm{C}^{\mathrm{k} N O T}\right\}[16$ and the set $\mathcal{T}$ is enough to realize any permutation on $k$ indices. As $\mathcal{T}$ exactly consists of the gates with graphical rules from above, we can state: 


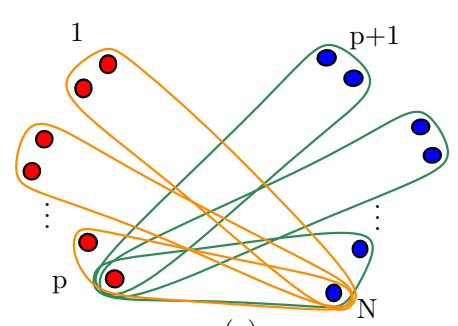

(a)

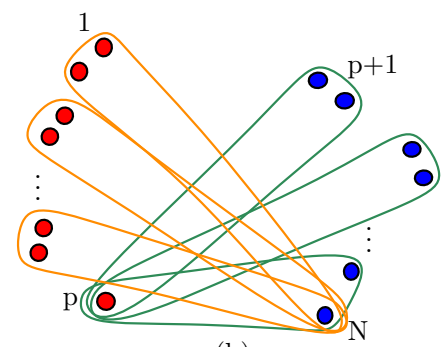

(b)

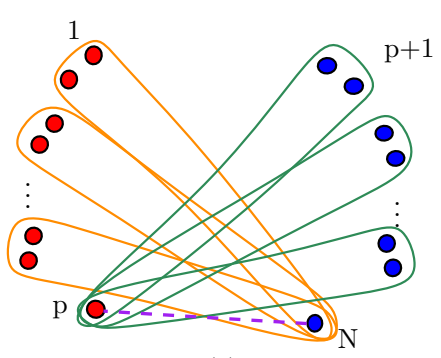

(c)

Figure 3. Different possibilities of the normal form for complete three-uniform hypergraph states. (a) The normal form if $p$ is even and $N$ is even. (b) The normal form if $p$ is odd and $N$ is odd. (c) The normal form if $p$ is odd and $N$ is even. We have two cases: (c1) The hypergraph without the edge $\{p, N\}$. This is the normal form if either both $(p+1)=2 \bmod 4$ and $(N-p+1)=2 \bmod 4$ or if $\operatorname{both}(p+1)=0$ $\bmod 4$ and $(N-p+1)=0 \bmod 4$. (c2) The hypergraph with the edge $\{p, N\}$. This is the normal form if $(p+1)=0 \bmod 4$ and $(N-p+1)=2 \bmod 4$ or if $(p+1)=2$ $\bmod 4$ and $(N-p+1)=0 \bmod 4$. The edge $\{p, N\}$ is represented by a dashed line.

Corollary 4. Every permutation unitary maps a hypergraph state to a hypergraph state and its graphical action can be seen as a composition of rules from $\mathcal{T}=$ $\left\{\mathrm{C}^{0} \mathrm{NOT}, \mathrm{CNOT}, \ldots, \mathrm{C}^{\mathrm{k}} \mathrm{NOT}\right\}$ graphical rules.

It is interesting to note how the different rules change the cardinality of edges. If $c$ is the cardinality of the largest edge in the hypergraph, the NOT gate can only create/erase edges with a cardinality strictly smaller then $c$. The CNOT gate can create/erase edges with cardinality smaller or equal to $c$, but the CCNOT can create edges with cardinality higher then $c$.

Finally, we demonstrate that the rule for the CNOT gate has a direct application: Consider a complete three-uniform hypergraph states, that is, the hypergraph contains all possible three-edges, but nothing else. These states can be thought as generalizations of GHZ states and violate Bell inequalities in a robust manner [6]. If one considers a possible bipartition $1, \ldots, p \mid p+1, \ldots, N$ of the particles, one may ask how the hypergraph can be simplified using unitaries that are local with respect to this bipartition. The following Lemma provides an answer, and we will use this later for the construction of witnesses.

Lemma 5. Consider an $N$-qubit complete three-uniform hypergraph state and a bipartition $1, \ldots, p \mid p+1, \ldots, N$. Then, using only local actions with respect to this bipartition the hypergraph can be reduced to the form shown in Fig. 3. We call this form a normal form of complete three-uniform hypergraph state respecting the bipartition $1, \ldots, p \mid p+1, \ldots, N$.

Proof. The proof consist of an application of a sequence of CNOT gates on both sides of the bipartition. Details are given in the Appendix. 


\section{Construction of witnesses}

In this section we consider the construction of entanglement witnesses as an application of the results derived so far. More specifically, we construct tight witnesses for fullyconnected three-uniform hypergraph states. These states are of special interest, as it has been shown that they violate Bell inequalities with an exponentially increasing amount and the violation is robust against particle loss. The Bell inequalities can be used to prove that there is some entanglement in the state, but in this section we will focus on entanglement witnesses for genuine multiparticle entanglement.

An entanglement witness is an observable which has a non-negative expectation value for all separable states, thus, a negative expectation value signals the presence of entanglement. There are many ways to construct entanglement witnesses, see Ref. [17] for an overview. One possible way to design a witness for a general state $|\psi\rangle$ is to consider the following observable

$$
\mathcal{W}=\alpha \mathbb{1}-|\psi\rangle\langle\psi|
$$

where $\alpha$ is the maximal overlap between the state $|\psi\rangle$ and the pure biseparable states. This can be computed by the maximal squared Schmidt coefficient occurring when computing the Schmidt decomposition with respect to all bipartitions,

$$
\alpha=\max _{\text {bipartitions }}\left\{\max _{\lambda_{k}^{\mathrm{BP}}}\left\{\left[\lambda_{k}^{\mathrm{BP}}\right]^{2}\right\}\right\}
$$

For usual graph states the witness can be determined in the following way [18]: First, for any bipartition one can generate a Bell pair between the two parties by making only local operations with respect to this partition. During local operations, however, the maximal Schmidt coefficient can only increase. This proves directly that for any bipartition $\lambda_{k}^{\mathrm{BP}} \leq 1 / 2$, so $\mathcal{W}=\mathbb{1} / 2-|G\rangle\langle G|$ is a witness. Using a similar construction, we can estimate $\alpha$ and write down a witness for three-uniform hypergraph states. Note that this scheme of constructing witnesses has recently been extended to other hypergraph states 19 .

Theorem 6. For any three-uniform hypergraph state $\left|H_{N}^{3}\right\rangle$ the operator

$$
\mathcal{W}=\frac{3}{4} \mathbb{1}-\left|H_{N}^{3}\right\rangle\left\langle H_{N}^{3}\right|
$$

is an entanglement witness detecting this state.

Proof. The proof is similar to the one for graph states, but in this case the aim is to share a the three-qubit hypergraph state between the bipartition. For this state the maximal squared Schmidt coefficient is $\alpha=3 / 4$. Given an $N$-qubit three-uniform state we consider a bipartition $1, \ldots, p \mid p+1, \ldots, N$. One can get rid of any edge which entirely belongs to either side of the bipartition. Since the graph is assumed to be connected at least one three-edge remains shared between the two parts. Without loss of generality 
we can assume that this edge is $e=\{p-1, p, p+1\}$. Now by making measurements in the Pauli-Z basis on every qubit except these three in $e$ we can disentangle all the qubits from the main hypergraph except $\{p-1, p, p+1\}$. For all possible measurement results, i.e. with probability one the resulting state is, up to local unitarians, a three-qubit hypergraph state consisting only of the edge $e$.

The previous witness can be used for any connected three-uniform hypergraph state, but is it no necessarily tight. For the special case of complete three-uniform states, where any possible three-edge is present, we derive a better witness in the following. Since this state is symmetric, the Schmidt coefficients depend only on the size of the partitions.

Lemma 7. Consider an N-qubit complete three-uniform hypergraph state. Then, the maximal squared Schmidt coefficient with respect to the bipartition 1 vs. $N-1$ qubits, is

$$
\begin{aligned}
& \lambda_{1}=\frac{1}{2} \text { if } N=4 k, \\
& \lambda_{1}=\frac{1}{2}+\frac{1}{2^{(N+1) / 2}} \text { if } N=4 k+1 \text { or } N=4 k+3, \\
& \lambda_{1}=\frac{1}{2}+\frac{1}{2^{N / 2}} \text { if } N=4 k+2 .
\end{aligned}
$$

For the 2 vs. $N-2$ partition it is $\lambda_{2}=\frac{1}{8}\left(3+\frac{\sqrt{2^{N+6}+4^{N}}}{2^{N}}\right)$. For the 3 vs. $N-3$ partition it is given by $\lambda_{3}=\frac{9}{16}$ if $N=6$ and for $N>6$ one has $\lambda_{3}<\frac{1}{2}$.

Proof. The proof is done by tracing out the parties and calculating the Schmidt coefficients as eigenvalues of the reduced states. Details can be found in the Appendix.

Theorem 8. An improved witness for the $N$-qubit complete three-uniform hypergraph state $\left|H_{N}^{\mathrm{c} 3}\right\rangle$ is given by

$$
\mathcal{W}=\alpha \mathbb{1}-\left|H_{N}^{\mathrm{c} 3}\right\rangle\left\langle H_{N}^{\mathrm{c} 3}\right|
$$

where $\alpha=\max \left\{\lambda_{1}, \lambda_{2}\right\}$.

Proof. We have to show that in general it is sufficient to consider the 1 vs. $N-1$ and 2 vs. $N-2$ partitions. First, the 3 vs. $N-3$ give only smaller Schmidt coefficients, as can be seen from the Lemma 7. For any other $1, \ldots, p \mid(p+1), \ldots, N$ bipartition with $p>3$ we use the normal form in Fig. 3 . If a resulting hypergraph is reduced either to Fig. 3 (b) or (c) [without the dashed edge], then on qubits $1 \ldots(p-3)$ the measurements in the Pauli-Z basis can be made. As a result, the hypergraph state $(p-2),(p-1), p \mid(p+1) \ldots N$ is obtained. We know from the Lemma 7 that the 3 vs. $N-3$ partition has a largest squared Schmidt coefficient less than $1 / 2$ (unless $N=6)$. Keeping in mind that measurements can never decrease the squared maximal Schmidt coefficient, we reach the conclusion that the bipartition $1, \ldots, p \mid(p+1), \ldots, N$ cannot contribute to the maximal Schmidt coefficient when $p \geq 3$. If in the normal form in Fig. 3 (c) the dashed edge is present, one can make measurements on both sides of 

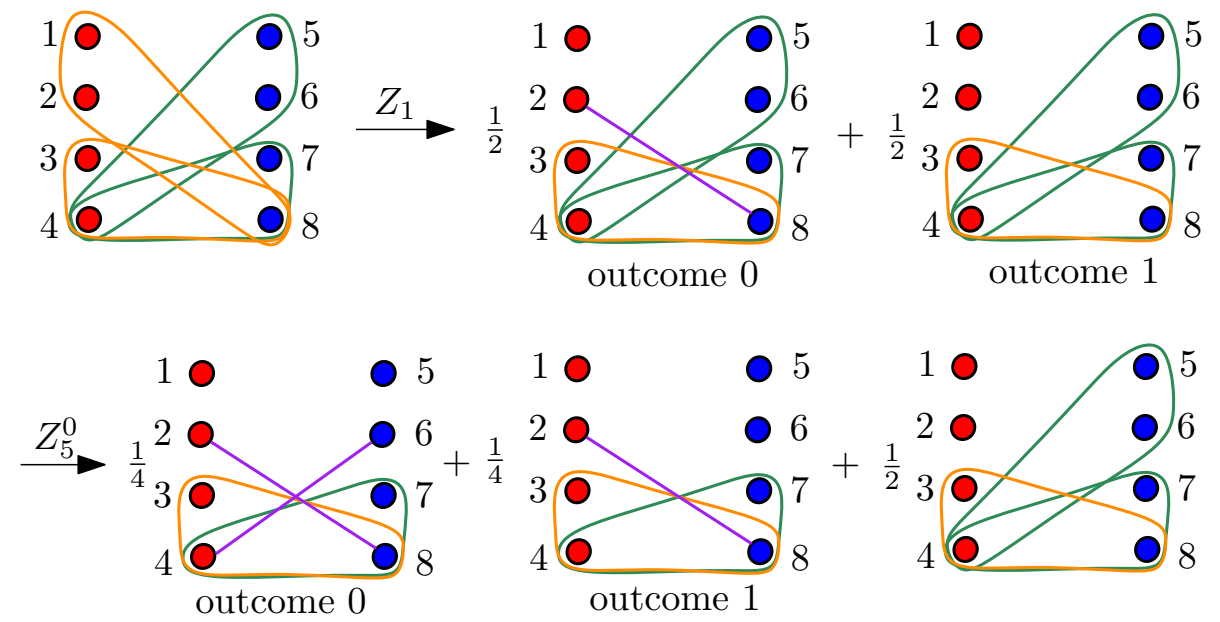

Figure 4. Estimation of the Schmidt coefficient for a 4 vs. 4 bipartition. See the text for further details.

the partition to reduce the state to a Bell state between qubits $p$ and $N$. This clearly gives a squared Schmidt coefficient $\lambda \leq 1 / 2$.

The final case is the state with a normal form in Fig. 3 (a). Here the strategy is as follows: Pauli-Z measurements are made on every qubit but eight of them, namely the qubits $p-3, p-2, \ldots, p+3, p+4$ remain untouched. This leaves us with the state given in Fig. 4, where the qubits have been relabeled. Then, a Pauli Z measurement is made on qubit 1 . With probability $1 / 2$ (in case of outcome 0 ), the edge $\{2,8\}$ is introduced and qubit 1 is disentangled. With probability $1 / 2$ (outcome 1 ) both qubits 1 and 2 are disentangled. For the first case (0 outcome), we again make a Pauli-Z measurement on qubit 5 , denoted by $Z_{5}^{0}$. This itself gives two possible outcomes with half-half probabilities, the outcome 0 gives the edge $\{4,6\}$ and disentangles qubit 5 and the outcome 0 disentangles qubits 4 and 5. Putting all measurement outcomes together with corresponding probabilities yields as a bound on the Schmidt coefficient

$$
\lambda \leq \frac{1}{4} \cdot \frac{1}{4}+\frac{1}{4} \cdot \frac{1}{2} \cdot \frac{1}{8}(3+\sqrt{5})+\frac{1}{2} \cdot \frac{1}{8}(3+\sqrt{2}) \approx 0.420202<\frac{1}{2} .
$$

Note that in this estimation it was used that one minus the largest squared Schmidt coefficient can be viewed as the geometric measure of entanglement for this partition, and this measure decreases under local operations even for mixed states.

\section{Conclusions}

In summary, we have extended the local complementation rule from graph states to hypergraph states. We also described the action of different gates on hypergraph states with graphical rules. Already for five qubits we showed with a simple example that local Pauli operations only are not enough to exhaust all local unitary equivalence classes of hypergraph states. Based on the rule for the CNOT gate, we developed a normal form 
for bipartitions of complete three-uniform hypergraph states. Based on this, we derived entanglement witnesses for these states.

There are several directions in which our work can be extended. First, it would be highly desirable to develop a general theory for entanglement witnesses for hypergraph states, similar to the existing theory for graph states [17]. Here, notions of the coulourability of a hypergraph may be developed to characterize how many measurements are needed to estimate the fidelity of a state. All this can help to observe hypergraph states experimentally. Another interesting question concerns the extent to which hypergraph states and their correlations can be simulated classically in an efficient manner. Our findings show that certain unitary operations have a graphical interpretation. This may be useful to decide whether their classical simulation is feasible. For graph states, the Gottesman-Knill theorem characterizes a set of operations that can be simulated efficiently and it would be highly desirable to identify similar operations for hypergraph states.

We thank Cornelia Spee for discussions. This work has been supported by the DFG and the ERC (Consolidator Grant 683107/TempoQ). Additionally, MG would like to acknowledge funding from the Gesellschaft der Freunde und Förderer der Universität Siegen.

\section{Appendix}

\subsection{Reduction of three uniform hypergraph states to the normal form in Lemma 5}

We prove Lemma 5 by considering first simple bipartitions, where the strategy of the proof is easier to explain. The proof for the general case then follows the same lines.

Lemma 9. Consider the bipartition $1 \mid 2,3, \ldots, N$ for an $N$-qubit complete three-uniform hypergraph state. Then this state is locally (for the given bipartition) equivalent to the three-uniform hypergraph state where every vertex is contained in only one edge and edges are of the form: $E=\{\{1, i, i+1\} \mid 2 \leq i<N$ and $i$ is even. $\}$ And only if $N=4 k$, an additional cardinality two edge appears, which is $\{1, N\}$.

Proof. Fig. 5 (a) represents the goal hypergraph state respecting a bipartition $1 \mid 2,3 \ldots N$. The algorithm to achieve this state is as follows:

(a) Erase all the edges which only contain subsets of vertices $\{2,3, \ldots N\}$. This operation is local with respect to the bipartition.

All the remaining edges are $\{\{1, i, j\} \mid i<j, 2 \leq i, j \leq N\}$.

(b) Apply $C N O T_{i, i+1}$, where $2 \leq i<N$.

To give an example, we start with the $\mathrm{CNOT}_{23}$ gate. The adjacency of 3 is $\mathcal{A}(3)=\{1, i\}$, where $i \neq 3,2 \leq i \leq N$. The edges introduced by the $C_{N O T_{23}}$ gate are $\left\{e_{t} \cup\{2\} \mid e_{t} \in \mathcal{A}(3)\right\}$ and therefore, this action removes all the edges where 2 is contained except the edge $\{1,2,3\}$ and adds the cardinality two edge $\{1,2\}$.

At this step the remaining edges are $\{\{1,2\},\{1,2,3\},\{1, i, j\} \mid i<j, 3 \leq i, j \leq N\}$. 
Graphical description of unitary transformations on hypergraph states

Since $2 \notin \mathcal{A}(i+1), i \geq 3$, it is clear that consecutive CNOT gates presented in this step do not modify edges containing 2. CNOT 34 erases all the edges where 3 is presented except already established $\{1,2,3\}$ and the gate where vertices 3 and 4 are presented together $\{1,3,4\}$. It also adds the cardinality two edge $\{1,3\}$. Repeating this procedure:

All the remaining edges are of the form $\{\{1, j, j+1\} \mid 2 \leq j<N\}$ and cardinality two edges $\{\{1, i\} \mid 2 \leq i<N\}$.

(c) Apply $C N O T_{i+2, i}$, where $2 \leq i<N-1$ and $i$ is even.

The adjacency of $i=2 \bmod 4$ right before applying the $C N O T_{i+2, i}$ gate is $\mathcal{A}(i)=\{\{1\},\{1, i+1\}\}$. The CNOT $T_{i+2, i}$ gate, therefore, erases/creates edges $\{\{1, i+2\},\{1, i+1, i+2\}\}$. This means that the adjacency of $i=0 \bmod 4$ is only $\{1, i+1\}$, and the $C N O T_{i+2, i}$ gate can only erase $\{1, i+1, i+2\}$. See Fig. 5 (b).

Here we have to consider several cases:

(1) $N$ is odd: All the remaining edges are of the form $\{1, i, i+1\}$, for even $2 \leq i \leq N$ and also $\{1, i\}$ for unless $i=0 \bmod 4$. It is easy to see that all two edges can be removed by action of Pauli-X's.

(2) $N$ is even: If $N=2 \bmod 4$, then the the last edge $\{1, N-1, N\}$ is erased and the edge $\{1, N\}$ cannot be created. Therefore, the last qubit is completely disentangled in this case. See Fig.5 (a).

(3) $N$ is even: If $N=0 \bmod 4$, then the the last edge $\{1, N-1, N\}$ is erased and the edge $\{1, N\}$ is created. See Fig. (b) for the exact procedure.

To sum up the previous theorem, there are three possibilities for the final hypergraph and it only depends on the number of parties in the hypergraph. If $N$ odd, then every vertex is exactly in one hyperedge. If $N=4 k$, then the final hypergraph corresponds to the one in Fig. 5 (a) including the dashed line. Note that this is in line with the fact that the maximal Schmidt coefficient for this case is $1 / 2$ (see Lemma 7), as there is a Bell pair shared across the bipartition [18]. In case $N=4 k+2$, the dashed line is missing, therefore, the last qubit can be removed and the result for the maximal Schmidt coefficient matches with $N=4 k+1$ case.

Lemma 10. Considering the bipartition $12 \mid 3,4, \ldots, N$ for an $N$-qubit fully-connected three-uniform hypergraph state. Then, this state is locally equivalent to the three-uniform hypergraph state already derived from the bipartition $2 \mid 3 \ldots N$ and in addition has the edge $\{1,2, N\}$.

Proof. The steps are very similar to the $1 \mid 23 \ldots N$ case:

(a) From a hypergraph we remove all the edges which do not contain either party 1, or 2.

All the remaining edges are $\{\{1, i, j\},\{2, i, j\},\{1,2, i\} \mid 2<i<j \leq N\}$ 


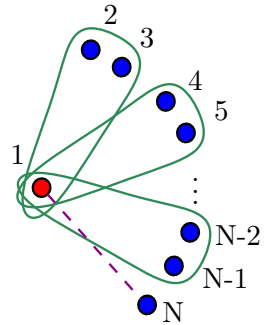

(a)
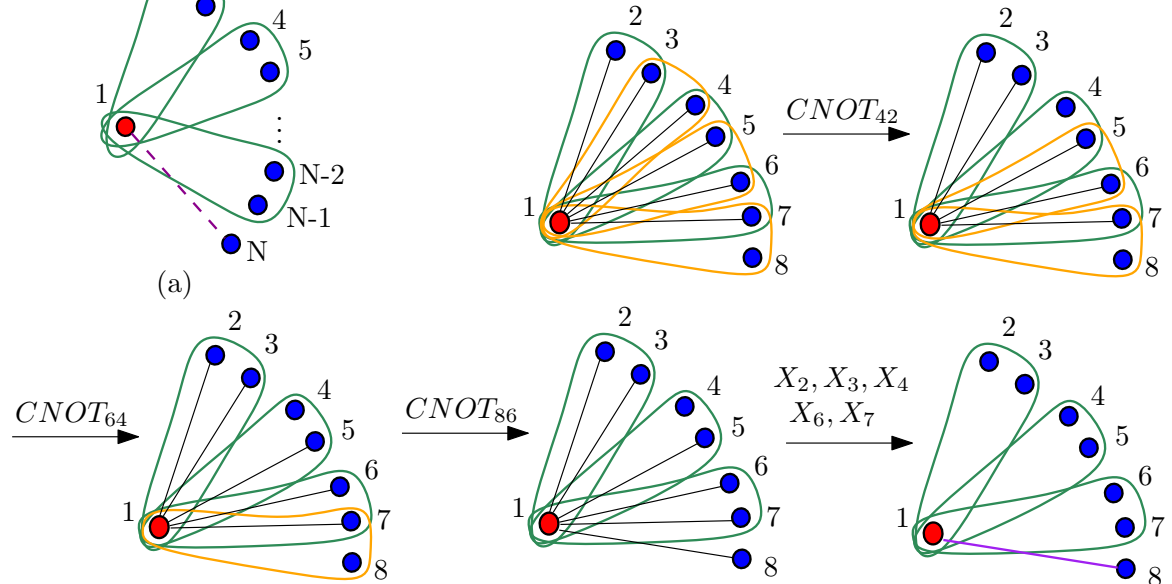

(b)

Figure 5. (a) The target graph for Lemma 9. (b) An example for step (c) of the proof of Lemma 9. See the text for further details.

(b) Apply $\mathrm{CNOT}_{12}$.

The adjacency of 2 is $\mathcal{A}(2)=\{\{i, j\},\{1, i\}\}$ for $2<i<j \leq N\}$. Augmented by the control qubit 1 , action of $C N O T_{12}$ removes $\{1, i, j\}$ and creates $\{1, i\}, 3 \leq i \leq N$. All the remaining edges are $\{\{1, i\},\{2, i, j\},\{1,2, i\} \mid 2<i<j \leq N\}$

(c) Apply $X_{2}$.

All the elements in the adjacency $\mathcal{A}(2)=\{\{1, i\},\{i, j\}\}$ for $2<i<j \leq N\}$ are added as edges to the hypergraph. Thus, all the edges of the type $\{1, i\}$ cancel out and $\{i, j\}$ can be directly removed.

All the remaining edges are $\{\{2, i, j\},\{1,2, i\} \mid 2<i<j \leq N\}$

(d) Apply the algorithm from Lemma 9 to the qubits $2 \mid 3 \ldots N$.

Each of the action of the $C N O T_{i, i+1}$ gate from Lemma 9 step (b), $3 \leq i<N$, removes the edge $\{1,2, i\}$ in addition to the its actions considered in Lemma 9 . Therefore only the edge $\{1,2, N\}$ remains of this type and other ones resulting from the action algorithm from Lemma 9 on qubits $2 \mid 3 \ldots N$.

Corollary 11. Considering any bipartition $1 \ldots p \mid(p+1) \ldots N$ of the complete threeuniform hypergraph state, the consecutively applying local CNOT gates (respecting the bipartition) reduces the hypergraph to the union of two hypergraphs as shown on Fig. 3. $p \mid p+1 \ldots N$ and $N \mid 1 \ldots p$, both already reduced to the normal form by the Lemma9.

This result is obtained by applying the algorithm from Lemma 9 first to $N \mid 1 \ldots p$ and then to $p \mid(p+1) \ldots N$. This ends the proof of Lemma 5 . 


\subsection{Proof of Lemma 7;}

Proof. First we consider 1 vs. $N-1$ bipartition. To calculate the maximal Schmidt coefficient we compute the reduced density matrix. As the state is symmetric, we only have to take the bipartition $1 \mid 2,3, \ldots, N$. We have

$$
\varrho_{1}=\operatorname{Tr}(|H\rangle\langle H|)_{2 \ldots N}=\frac{1}{2^{N}}\left(\begin{array}{cc}
2^{N-1} & a \\
a & 2^{N-1}
\end{array}\right) .
$$

The diagonal elements follow directly from the representation of the hypergraph state in Eq. (2) and do not depend on the structure of the hypergraph. For computing the off-diagonal entries, we write the hypergraph state as

$$
|H\rangle=|0\rangle \sum_{x}\left[(-1)^{f_{0}(x)}|x\rangle\right]+|1\rangle \sum_{x}\left[(-1)^{f_{1}(x)}|x\rangle\right] .
$$

with $x \in\{0,1\}^{(N-1)}$. Since we deal with three-uniform complete hypergraph states, we have $f_{0}=\left(\begin{array}{c}w(x) \\ 3\end{array}\right)$ and $f_{1}=\left(\begin{array}{c}w(x)+1 \\ 3\end{array}\right)$, where $w(x)$ is the weight (i.e., the number of " 1 " entries) of $x$. We can then write

$$
a=\sum_{x}(-1)^{f_{0}(x)+f_{1}(x)}
$$

The values of $f_{0}$ and $f_{1}$ do only depend on $w(x)$ mod 4 . Instead of summing over $x$, we can also sum over all possible $k=w(x)$ in Eq. (23) and distinguish the cases of $k$ mod 4 . The value for a given $k$ is then up to the sign given by the numbers of possible $x$ with the same $w(x)=k$. We have:

$$
\begin{aligned}
a & =\sum_{k=0,4 \ldots}^{2^{N-1}}\left[\left(\begin{array}{c}
N-1 \\
k
\end{array}\right)+\left(\begin{array}{c}
N-1 \\
k+1
\end{array}\right)-\left(\begin{array}{c}
N-1 \\
k+2
\end{array}\right)-\left(\begin{array}{c}
N-1 \\
k+3
\end{array}\right)\right] \\
& =\operatorname{Re}\left[(1+i)^{N-1}\right]+\operatorname{Im}\left[(1+i)^{N-1}\right] .
\end{aligned}
$$

To give the final result, we have to consider several cases in Eq. 24): If $N=4 \ell$, then $a=0$, therefore, $\varrho_{1}$ is maximally mixed and $\lambda_{1}=1 / 2$. If $N=4 \ell+1$ or $N=4 \ell+3$, then $a= \pm 2^{\frac{N-1}{2}}$. Then, it follows that $\lambda_{1}=1 / 2+1 / 2^{\frac{N+1}{2}}$. Similarly, for $N=4 \ell+2$, $a= \pm 2^{\frac{N}{2}}$ and therefore $\lambda_{1}=1 / 2+1 / 2^{\frac{N}{2}}$. This ends the computation of $\lambda_{1}$.

Second, we look at the 2 vs. $N-2$ bipartitions. The idea of the proof very much resembles the previous case. First, we take the bipartition $1,2 \mid 3,4, \ldots, N$ and trace out the second part:

$$
\varrho_{12}=\operatorname{Tr}(|H\rangle\langle H|)_{3 \ldots N}=\frac{1}{2^{N}}\left(\begin{array}{cccc}
2^{N-2} & a_{+} & a_{+} & 0 \\
a_{+} & 2^{N-2} & 2^{N-2} & a_{-} \\
a_{+} & 2^{N-2} & 2^{N-2} & a_{-} \\
0 & a_{-} & a_{-} & 2^{N-2}
\end{array}\right) .
$$


For computing the entries, we express a hypergraph state in the following way:

$$
\begin{aligned}
|H\rangle & =|00\rangle \sum_{x}\left[(-1)^{f_{00}(x)}|x\rangle\right]+|01\rangle \sum_{x}\left[(-1)^{f_{01}(x)}|x\rangle\right] \\
& +|10\rangle \sum_{x}\left[(-1)^{f_{10}(x)}|x\rangle\right]+|11\rangle \sum_{x}\left[(-1)^{f_{11}(x)}|x\rangle\right] .
\end{aligned}
$$

with $x \in\{0,1\}^{(N-2)}$. The diagonal elements of $\varrho_{12}$ are, as before, easy to determine. This is also the case for the two anti-diagonal terms $|01\rangle\langle 10|=| 10\rangle\langle 01|$ as $f_{01}+f_{10}$ is always even. The next term, $a_{+}$, is derived as Eqs. 23, 24): $a_{+}=\operatorname{Re}\left[(1+i)^{N-2}\right]+\operatorname{Im}\left[(1+i)^{N-2}\right]$. For the term $|00\rangle\langle 11|, f_{00}(x)+f_{11}(x)$ is even if $w(x)$ is even and is odd if $w(x)$ is odd. Therefore $\sum_{x}(-1)^{f_{00}+f_{11}}=0$. For the last term we find $a_{-}=a-a_{+}=$ $\operatorname{Re}\left[(1+i)^{N-2}\right]-\operatorname{Im}\left[(1+i)^{N-2}\right]$.

Putting all these terms together in the matrix, one can calculate the maximal eigenvalue of $\varrho_{12}$ :

$$
\begin{aligned}
\lambda_{2} & =\frac{1}{8}\left(3+\frac{\sqrt{4^{N}+128\left(a_{+}^{2}+a_{-}^{2}\right)}}{2^{N}}\right)=\frac{1}{8}\left(3+\frac{\sqrt{4^{N}+64 A b s\left[(1+i)^{N}\right]^{2}}}{2^{N}}\right) \\
& =\frac{1}{8}\left(3+\frac{\sqrt{4^{N}+2^{N+6}}}{2^{N}}\right) .
\end{aligned}
$$

Finally, we have to consider the $1,2,3 \mid 4 \ldots N$ bipartition and write down the reduced density matrix:

$$
\varrho_{123}=\operatorname{Tr}(|H\rangle\langle H|)_{4 \ldots N}=\frac{1}{2^{N}}\left(\begin{array}{ccccccccc}
2^{N-3} & c & c & 0 & c & 0 & 0 & b \\
c & 2^{N-3} & 2^{N-3} & -b & 2^{N-3} & -b & -b & 0 \\
c & 2^{N-3} & 2^{N-3} & -b & 2^{N-3} & -b & -b & 0 \\
0 & -b & -b & 2^{N-3} & -b & 2^{N-3} & 2^{N-3} & -c \\
c & 2^{N-3} & 2^{N-3} & -b & 2^{N-3} & -b & -b & 0 \\
0 & -b & -b & 2^{N-3} & -b & 2^{N-3} & 2^{N-3} & -c \\
0 & -b & -b & 2^{N-3} & -b & 2^{N-3} & 2^{N-3} & -c \\
b & 0 & 0 & -c & 0 & -c & -c & 2^{N-3}
\end{array}\right),
$$

where $c=\operatorname{Re}\left[(1+i)^{N-3}\right]-\operatorname{Im}\left[(1+i)^{N-3}\right]$ and $b=-\operatorname{Re}\left[(1+i)^{N-3}\right]+\operatorname{Im}\left[(1+i)^{N-3}\right]$.

From this we can be derive all possible values of maximal Schmidt coefficient $\lambda_{3}$. If $N \equiv 4 k$, then $\lambda_{3}=2^{-2}+2^{-4 k-3} \sqrt{48 \cdot 2^{4 k}+4^{4 k}}$. If $N \equiv 4 k+1$, then $\lambda_{3}=2^{-2}+2^{-2 k-1}+2^{-4 k-3} \sqrt{16^{k}\left(16+2^{4 k}+2^{2 k+2}\right)}$. If $N \equiv 4 k+2$, then $\lambda_{3}=$ $2^{-2}+2^{-2 k-1}+2^{-4 k-3} \sqrt{2^{4 k}\left(2+2^{2 k}\right)^{2}}$ and finally, if $N \equiv 4 k+3$, then $\lambda_{3}=2^{-2}+$ $2^{-2 k-2}+2^{-4 k-3} \sqrt{2^{4 k}\left(4+2^{4 k}+2^{2 k+1}\right)}$. It can be easily seen that $\lambda_{3}$ is decreasing with $N$ and it is only greater than $1 / 2$ when $N=6$.

\section{References}

[1] M. Hein, W. Dür, J. Eisert, R. Raussendorf, M. Van den Nest, and H.-J. Briegel, Entanglement in Graph States and its Applications, in Quantum Computers, Algorithms and Chaos, edited 
by G. Casati, D.L. Shepelyansky, P. Zoller, and G. Benenti (IOS Press, Amsterdam, 2006), quant-ph/0602096.

[2] C. Kruszynska and B. Kraus, Phys. Rev. A 79, 052304 (2009).

[3] M. Rossi, M. Huber, D. Bruß, and C. Macchiavello, New J. Phys. 15, 113022 (2013).

[4] R. Qu, J. Wang, Z. Li, and Y. Bao, Phys. Rev. A 87, 022311 (2013).

[5] O. Gühne, M. Cuquet, F. E. S. Steinhoff, T. Moroder, M. Rossi, D. Bruß, B. Kraus, and C. Macchiavello, J. Phys. A: Math. Theor. 47, 335303 (2014).

[6] M. Gachechiladze, C. Budroni, and O. Gühne, Phys. Rev. Lett. 116, 070401 (2016).

[7] M. Rossi, D. Bruß, and C. Macchiavello, Phys. Scr. T160, 014036 (2014).

[8] J. Miller and A Miyake, npj Quantum Information 2, 16036 (2016).

[9] M. van den Nest, J. Dehaene and B. De Moor, Phys. Rev. A 69, 022316 (2004).

[10] A. Cabello, A.J. Lopez-Tarrida, P. Moreno, and J.R. Portillo, Phys. Rev. A 80, 012102 (2009).

[11] Z. Ji, J. Chen, Z. Wei, and M. Ying, Quantum Inf. Comp. 10, 97 (2010).

[12] N. Tsimakuridze and O. Gühne, J. Phys. A: Math. Theor. 50, 195302 (2017).

[13] X.-Y. Chen and L. Wang, J. Phys. A: Math. Theor. 47, 415304 (2014).

[14] G.W. Leibniz, private notes, Niedersächsische Landesbibliothek Hannover, LH XXXV 4.8 f.1-2 (1678).

[15] T. Toffoli, "Reversible computing," in Lecture Notes in Computer Science, Vol. 84 (Springer, 1980), pp. 632-644.

[16] S. Xu, arXiv:1506.03777.

[17] O. Gühne and G. Tóth, Phys. Rep. 474, 1 (2009).

[18] G. Tóth and O. Gühne, Phys. Rev. Lett. 94, 060501 (2005).

[19] M. Ghio, D. Malpetti, M. Rossi, D. Bruß, and C. Macchiavello, arXiv:1703.00429. 